Journal of

Clinical and Anatomic Pathology

\title{
The Malignant Transformation of Oral Lichen Planus and Oral Lichenoid Le- sions, a Case Report and Review of the Literature
}

\section{Selene Saraf', Nadereh Ghanee DMD, Mary Nathanson Kilo MD, Abeer Alkhalidi DMD}

Pacific Northwest Kaiser Dental, U.S.A

*Corresponding author: Selene Saraf, Pacific Northwest Kaiser Dental, U.S.A; E-mail: selenesaraf@gmail.com

Received Date: April 09, 2019 Accepted Date: May 06, 2019 Published Date: May 08, 2019

Citation: Selene Saraf (2019) The Malignant Transformation of Oral Lichen Planus and Oral Lichenoid Lesions, a Case Report and Review of the Literature. J Clin Anat Pathol 4: 1-6.

\section{Abstract}

Oral lichen planus (OLP) is a chronic immunological disorder of unknown etiology involving skin and mucosa. Oral lichenoid lesions (OLL) are clinically and histologically similar to OLP. OLL has also been described as an oral lichenoid reaction or lichenoid contact stomatitis. The etiology of OLL is considered to be an exogenous factor such as oral hygiene products, food, medications or dental material. It is not a simple task to distinguish between the OLP and OLL. Here we report a case of OLL with malignant transformation that occurred two times. Diagnostic criteria to distinguish these two entities will be discussed as well.

\section{Introduction}

Oral lichen planus (OLP) and oral lichenoid lesions (OLL) have generated a lot of debate in the literature. In contrast to the unknown nature of OLP, OLL is often associated with a known inciting factor. "Oral lichenoid lesions" are also called, "oral drug reactions," "oral lichenoid reactions," and lichenoid contact stomatitis." Malignant transformation of both entities has been reported. Most studies have shown OLL to have a slightly higher potential of malignant transformation compared to OLP.

\section{Case Report}

A 64-year old female patient was seen in a Pacific Northwest Kaiser Dental, oral pathology clinic for recurrent oral ulcerations in November 2012. The patient's medical history included seasonal allergies, depression, hypothyroidism, mild psoriasis, and related medications. The patient was never a smoker. The head, neck, and oral exam revealed moderate dryness of the oral mucosa, erythema, inflammation, and keratinization only concentrated within the upper gingiva. She stated that a previous physician diagnosed her with lichen planus. However, her clinical features suggested otherwise and did not show typical OLP appearance. The patient complained about a generalized burning sensation with

C2019 The Authors. Published by the JScholar under the terms of the Creative Commons Attribution License http://creativecommons.org/licenses/ by/3.0/, which permits unrestricted use, provided the original author and source are credited. certain foods. A cytology oral smear was done and the result was, "strongly positive for candidiasis." Based on clinical features, the diagnosis was OLL with superimposed candidiasis. However, OLP was still considered. Diet analysis was done and oral hygiene products were reviewed. Since some features were suggestive of OLL, possible irritants to the oral mucosa were discussed and a new diet plan was recommended. She was prescribed antifungal rinse and Lidex $0.05 \%$ as a topical steroid. The patient was instructed to practice this regimen twice daily and taper off after signs and symptoms improve.

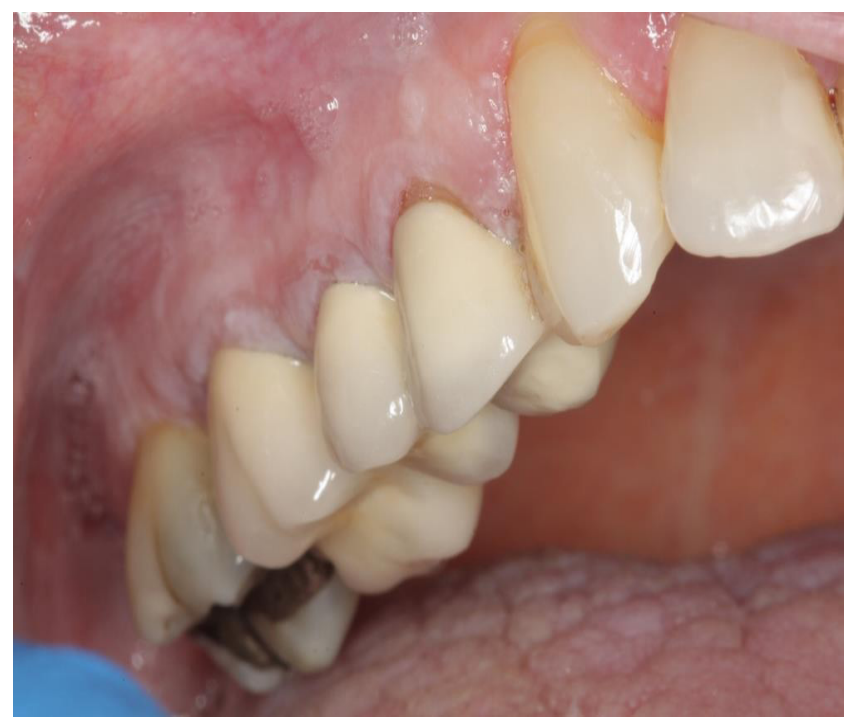

Figure 1. Upper right gingiva showing keratotic features, 2012 


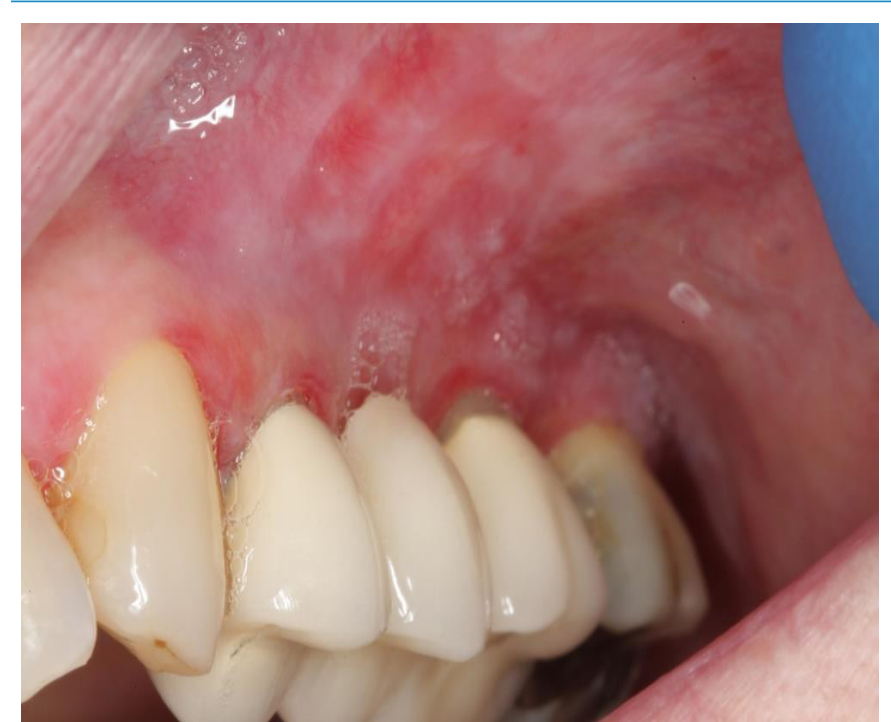

Figure 2. Upper left gingiva showing diffused erythema, symptomatic, 2012

The patient returned after two months and was happy with her improved results. From then, she continued her treatment and check-ups through Kaiser Medical due to better coverage. She was periodically checked by an ENT doctor. She returned to Kaiser's oral pathology clinic in July, 2015 because of a severe flare-up of the affected area. The patient emphasized maintaining the same regimen she was given in 2012 which included diet, oral hygiene instructions, and applications of the Lidex topical steroid.

Another cytology was done and was again, strongly positive for candida. Oral mucosa still showed a variable mixture of erythema, keratinization, and inflammation mostly involving the gingiva. Features were slightly more prominent compared to 2012 and symptoms were significantly worse. Antifungal rinse was added to the treatment and the patient was scheduled for a re-check in two months.

Periodic phone appointments in which she reported improved results occurred within those two months as well. In September 2015 she returned for a re-check and the exam showed diffuse erythema with small white, new keratotic growth on the upper right gingiva. This area was previously thick and keratotic with no erythema. (See picture from 2012).

That area was surgically removed and was submitted to the pathology lab. The result was squamous cell carcinoma, invasive, with an estimated depth of invasion at 5 millimeters. The patient was referred to Kaiser's ENT team. Partial maxillectomy was done and the pathology result showed negative margins. The P16 test was negative. An incisional biopsy of the buccal gingiva of number 24 was also done and showed lichenoid inflammation.

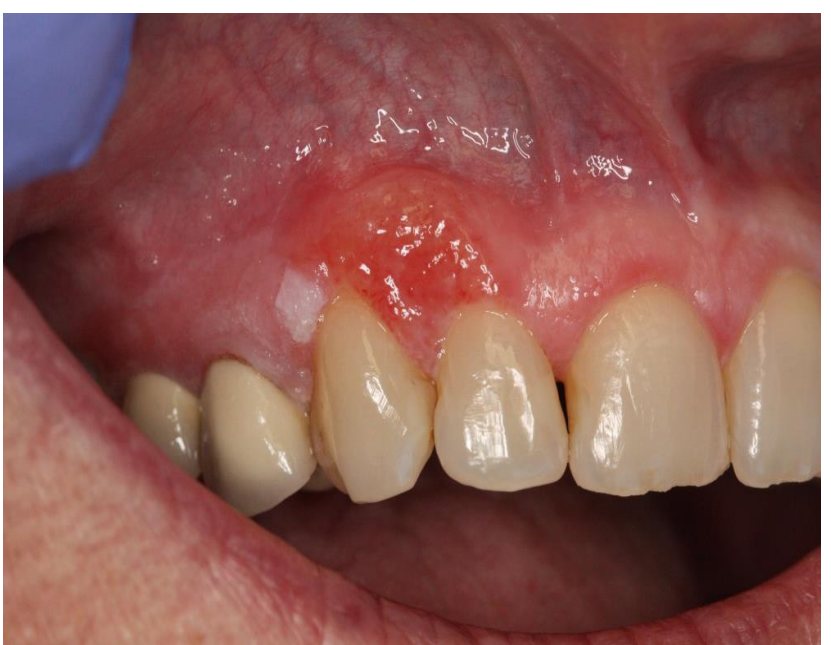

Figure 3. Upper right gingiva showing new features. Biopsy result showed squamous cell carcinoma. September, 2015

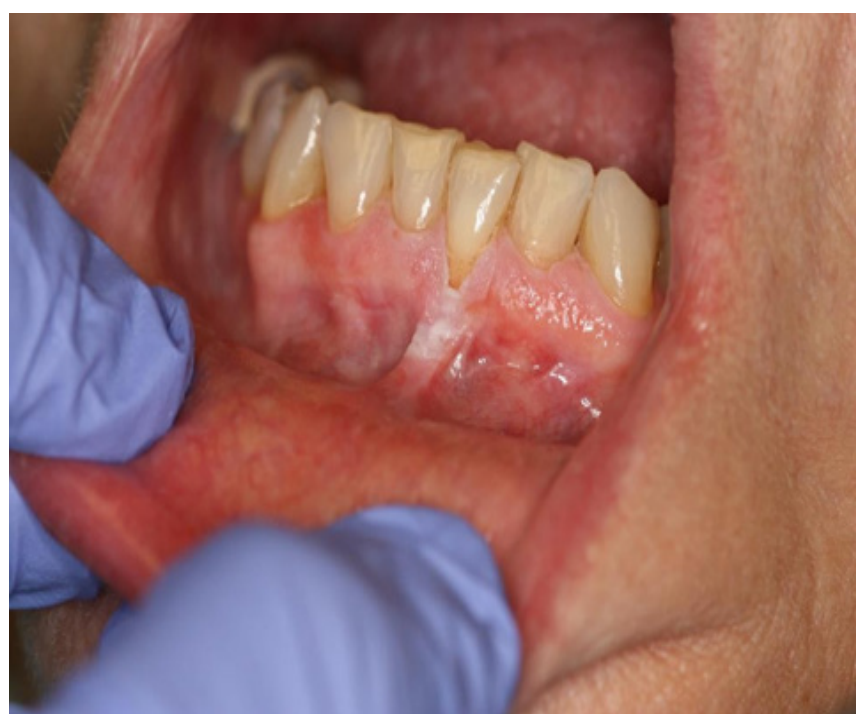

Figure 4. Incisional biopsy of buccal gingiva area, tooth \#24. Results were lichenoid reaction

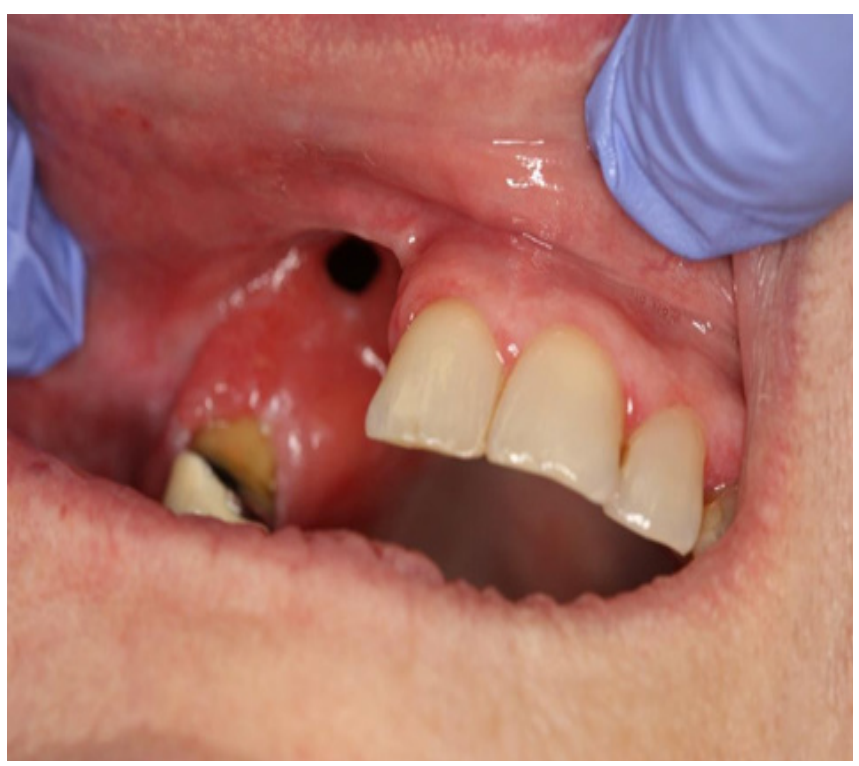

Figure 5. Surgery site, post-cancer removal 
The patient was then checked periodically at the oral pathology clinic. Her features remained mild and no major changes were noticed. However, repeated cytology exams were always positive for candida. Therefore, topical antifungal rinse was combined with topical steroid treatment. In September 2016 some areas such as buccal gingiva of \#19 and dorsal tongue showed keratotic changes. Dorsal tongue features were more suggestive of OLP at that point. The buccal of \#13 which always showed some degree of erythema and inflammation, appeared more inflamed, an incisional biopsy was done and the result was, "squamous hyperplasia with inflammation and fungal hyphae."

Periodic checks and close observations continued with the oral pathology clinic until September 2017. At that time, the area of previous cancer showed slight growth with erythema and inflammation, excisional biopsy was done and the result was, "lichenoid inflammation, negative for dysplasia." In October 2017 another cytology was positive for candida. In November of that year, a biopsy for buccal of \#24 was done and the result was, "lichenoid reaction." In March 2018, a biopsy of the upper right gingiva showed, "lichenoid inflammation with atypia." In June 2018 a biopsy of area \#24 was sent for direct immunofluorescence studies and was negative for fibrinogen at basement membrane zone. In September 2018, area of upper right gingiva showed new growth and the result of the biopsy was, "secondary invasive cancer, squamous cell carcinoma." In October 2018 the patient went through secondary cancer treatment with an ENT team. The result was," squamous cell carcinoma," and P16 was negative.

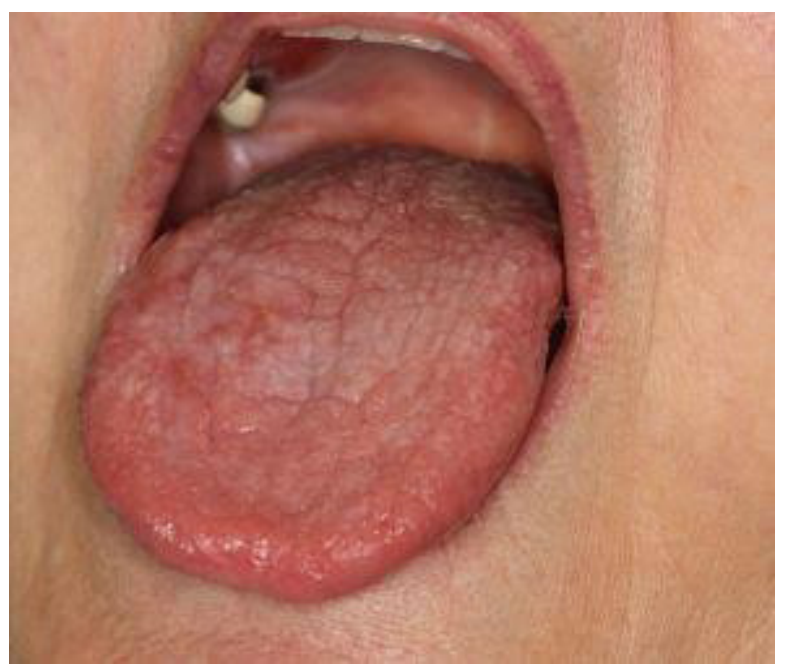

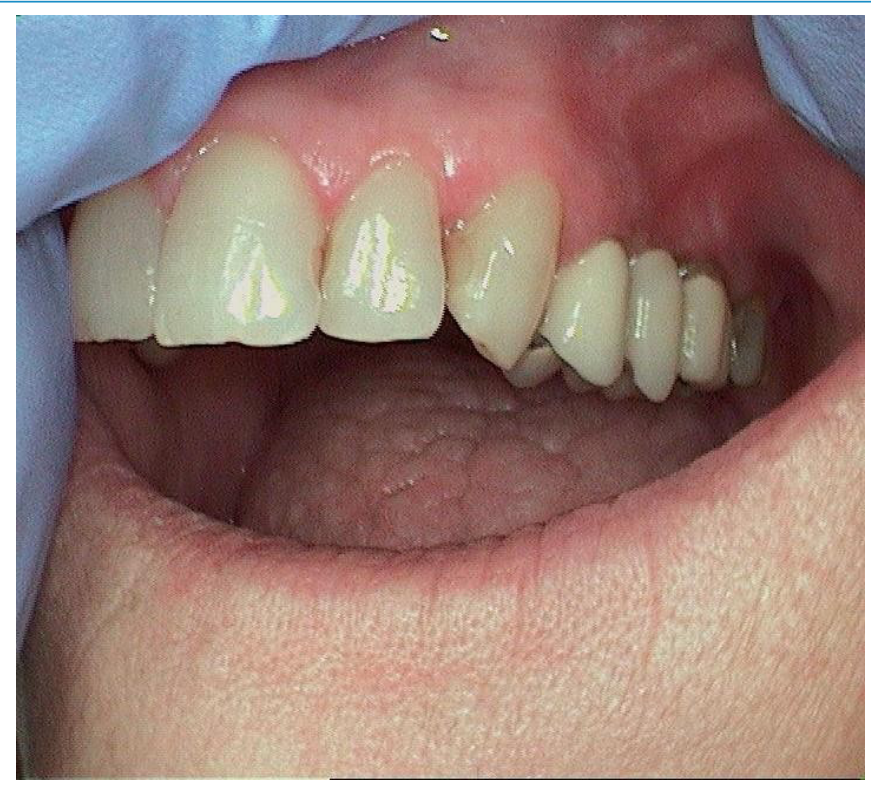

Figures 7

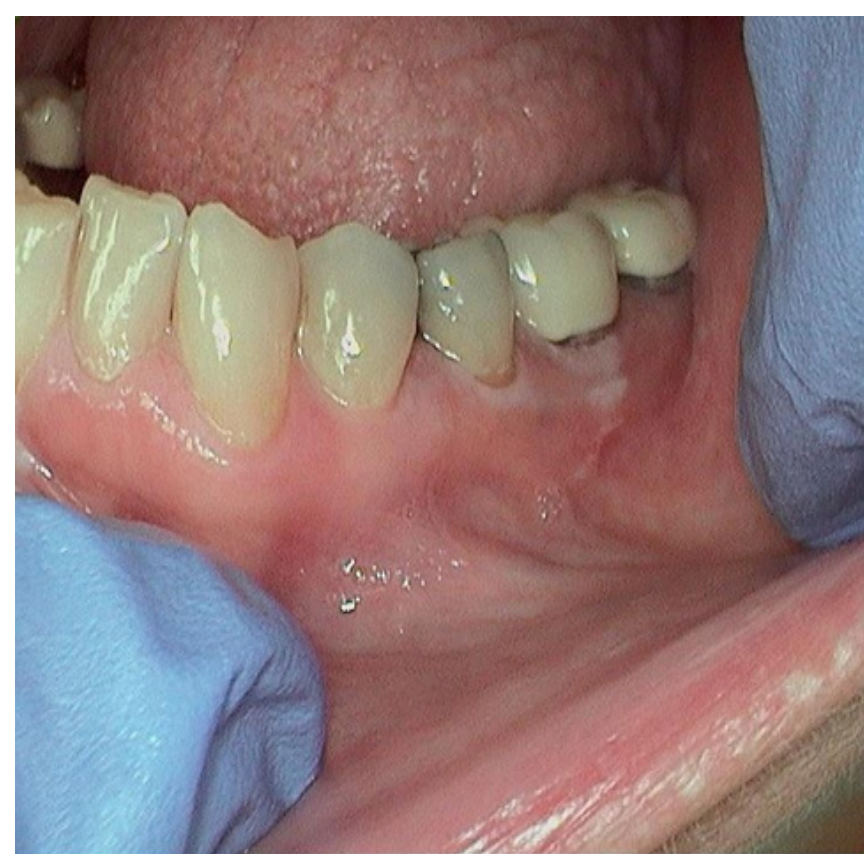

Figures 8

Figures 6-8. Clinical features changed on the dorsal tongue, buccal gingiva of \#19 and \#13. September 2016

Figures 6 


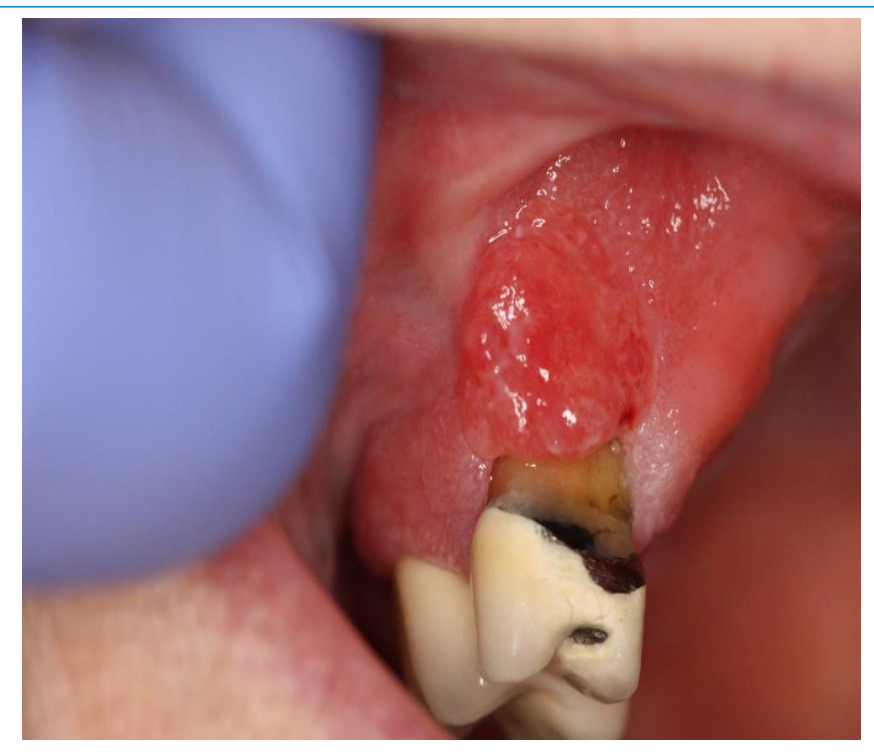

Figures 9

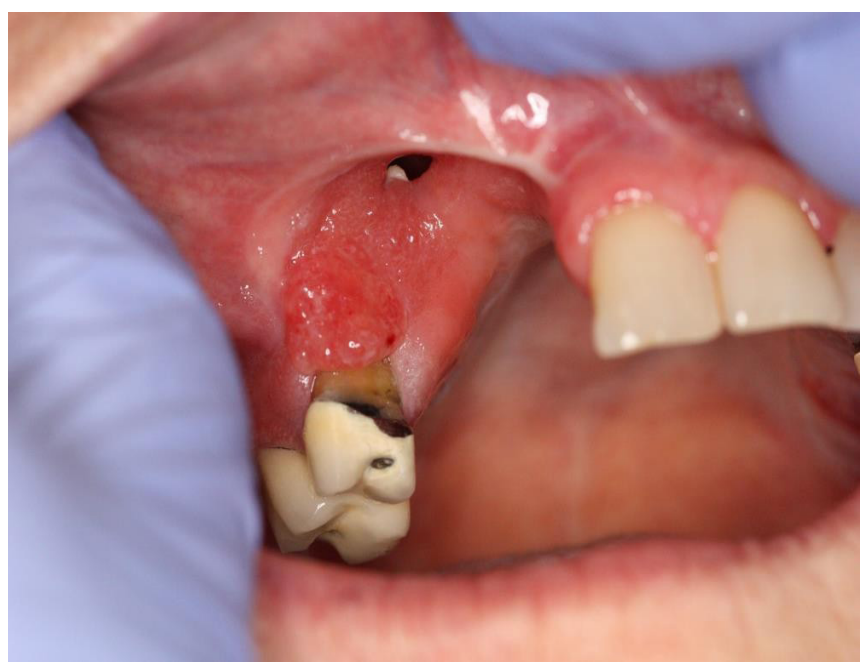

Figures 10

Figures 9\&10. Recurrent squamous cell carcinoma at previous surgical site in September, 2018

\section{Discussion}

OLP and OLL have similar clinical and histologic features. Often times, it is not an easy task to distinguish between the two. OLP is a chronic T cell-mediated disease of oral mucosa and skin. Clinical presentation of OLP usually has a symmetrical pattern, involving gingiva, dorsum of the tongue, labial mucosa and lower vermilion lip. 10\% of patients with OLP have features confined to the gingiva. [10]. Patients with OLP who present unilateral lesions, over time will develop multiples site of involvement [5].

Clinical features suggestive of OLL include atypical sites for OLP and dental restorations or a denture component in the vicinity of the lesions [7]. Certain medications can cause OLL including anti-hypertensive medications, Imatinib Mesylate, Zidovudine, and Carbamazepine [2]. In addition, dental materials also cause OLL symptoms. One example is hypersensitivity to mercury which eventually results to OLL [4]. Tartar, plaque and hyposalivation also cause OLL [11]. Correlation between OLL and GVHD has also been reported [5]. Some studies showed OLL occurring more on gingiva and palate, unlike OLP that occurs on buccal mucosa and tongue [11]. Both OLL and OLP can develop superimposed candidiasis which partially could be due to the chronic use of topical steroids.

In comparison, there is a lot of debate between OLP and OLL on a microscopic level. The microscopic view of OLP presents subepithelial dense infiltrations of lymphohistiocytic cells as well as features of the ruptured epithelial basement membrane. OLL will present similar features but it will have deeper infiltrations of inflammatory cells containing eosinophils, plasma cells, and neutrophils [8]. The World Health Organization (WHO) 1978 criteria for the diagnosis of OLP includes ortho or para keratinization, liquefaction degeneration of basal cell layer, a well-defined band like lymphocytic infiltration close to the basal layer and Civatte bodies in basal epithelium and lamina propria. [16]. One study found that $29 \%$ of epithelial dysplasia cases had a presence of lichenoid features. Therefore, enforcing the wrong treatment plan is possible due to similar band-like inflammatory cell infiltration and basal cell degeneration. This is also why a microscopic examination of the tissue is crucial.

Although distinguishing between OLL and OLP remains a controversial topic; The WHO set of diagnostic criteria that were devised in 1978 provides a substantial approach to the topic. The clinical features for OLL include the presence of atrophic lesions with or without erosion while OLP can be identified due to its bilateral, symmetrical lesions. In addition, both conditions can show skin eruptions. OLL and related skin eruptions can be resolved with the termination of the causative drug whereas OLP will not be affected by drug use [16].

Direct immunofluorescence studies are often done for oral and skin autoimmune disorders. Some studies have shown shaggy deposition of fibrinogen along the basement membrane zone in both OLP and OLL [3]. Fluorescence was also observed to be less intense in OLL [15]. On the other hand, indirect immunofluorescence studies are negative in OLP and may detect circulatory basal cell autoantibodies in the patient's serum [12]. However, overall immunofluorescence studies are not a reliable way to differentiate between OLL and OLP due to how similar the results could be [9].

Since OLP and OLL have malignant potential, and sometimes lesions can be caught at a dysplastic stage, the term oral lichenoid dysplasia (OLD) has been described. OLD microscopically shows dysplastic epithelium with lamina propria showing a lichenoid reaction. The definition of OLD has been controversial since it is not truly clear whether dysplasia or OLL/OLP is the primary pathology [18].

One question worth addressing is the correlation between psoriasis and OLP because the patient focused in this study has mild psoriasis. She has a strong family history of pso- 
riasis including a son with a severe and persistent form. There have not been many studies regarding the coexistence between OLP and psoriasis. However, one study monitored a 47-year old man who had OLP and psoriasis present throughout his body including his back, trunk, and thighs. He was given a twice-daily topical dexamethasone propionate ointment for the OLP and hydrocortisone and maxacalcitol ointments for psoriasis. Use of these medications caused the OLP to disappear after six months and the psoriasis was sporadic for the next two years. This study concluded that coexistence of OLP and psoriasis is rare yet still happens. Further research was done by the Peking Medical College in Beijing and they found that in a group of 1743 psoriasis patients, 10 reported to also have OLP. They also concluded that both are known to occur after patients have been given anti-immune necrosis factor (TNF) [14]. Overall, research still needs to be done regarding this topic.

In several studies, researchers have tried to find the causative factors of OLL by performing allergy skin patch tests. It is recommended but remains controversial [1]. Most studies show that OLL is more prone to malignant transformation than OLP [6]. In these studies, all OLL cases microscopically did not show band-like lymphocytic infiltration close to the basement membrane zone, Civatte bodies, or other features typically seen in OLP. Immunofluorescence studies did not show deposition of fibrinogen in the basement membrane zone.

In this case, in every follow-up appointment since clinical and histological features were suggestive of OLL, possible inciting factors were reviewed with the patient, including food, oral hygiene products, and dental material. Her medications did not change over the years that the patient was under follow-up and did not seem to be a causative factor. However, anti-depressants could be causative for OLL either directly or indirectly since they induce dry mouth. Another factor worth considering is that the patient was on hypothyroidism medication during the years of her follow-up. A group of oral pathologists in Finland did a study to address whether there was a correlation between the coexistence of OLP and hypothyroidism. Their results showed that $10 \%$ of their 152 OLP cases also had hypothyroidism. They concluded, however, that larger sample size and further observations needed to be done in order to confirm a correlation [17].

\section{Conclusion}

With chronic cases of OLP and OLL, it is crucial to keep the lesions and extensive inflammation under control. In both conditions, any causative factor including chemical, mechanical, or thermal trauma should be avoided. Regular follow-up, close observation, and multiple biopsies are recommended. Malignant transformation of OLP and OLL ranges between $0.5 \%$ to $3 \%$. The patient should be advised and informed regarding the malignant transformation of the condition. The patient should be told to be compliant to keep the inflammation and severity of the lesions under control.
The patient should be advised to come for regular check-ups due to the possibility of malignant transformation. This is the goal of all healthcare providers since early detection of squamous cell carcinoma is crucial.

\section{References}

1. Al-Hashimi, Schifter, Lockhart, Wray, Brennan, Migliorati, Axéll, Bruce, et al. (2007) "Oral Lichen Planus and Oral Lichenoid Lesions: Diagnostic and Therapeutic Considerations." Oral Surgery, Oral Medicine, Oral Pathology, Oral Radiology and Endodontology 103.3 S25.e1-25.e12.

2. Baris E, B Sengüven, T Tüzüner, and S E Gültekin (2014) "Oral Lichenoid Lesions Related to Drugs: Review of Clinicopathological Features and Differential Diagnosis." European Journal of Inflammation 12.2: 217-25.

3. Buajeeb, Waranun, Nis Okuma, Supanee Thanakun, and Titikarn Laothumthut (2015) "Direct Immunofluorescence in Oral Lichen Planus." Journal of Clinical and Diagnostic Research: JCDR 9.8:34-37.

4. Do Prado, Marocchio, and Filipino (2009) "Oral Lichen Planus versus Oral Lichenoid Reaction: Difficulties in the Diagnosis." Indian Journal of Dental Research 20.3: 361-64.

5. Dudhia, Bhavin, Dudhia, Sonal, Patel, et al. (2015) "Oral Lichen Planus to Oral Lichenoid Lesions: Evolution or Revolution." 19.3: 364-370.

6. Fitzpatrick, Hirsch, and Gordon (2014) "The Malignant Transformation of Oral Lichen Planus and Oral Lichenoid Lesions: A Systematic Review: A Systematic Review." The Journal of the American Dental Association 145.1: 45-56.

7. Ismail, Sumairi B, Kumar, Satish K S, et al. (2007) "Oral Lichen Planus and Lichenoid Reactions: Etiopathogenesis, Diagnosis, Management, and Malignant Transformation." Journal of Oral Science 49.2: 89-106.

8. Jahanshahi, Gholamreza, ParichehrGhalayani, and LalehMaleki (2012) "Mast Cells Distribution and Variations in Epithelium Thickness and Basement Membrane in Oral Lichen Planus Lesion and Oral Lichenoid Reaction." Dental Research Journal 9.2: 180-184.

9. Khudhur, Ahmed Salih, Giovanni Di Zenzo, and Marco Carrozzo (2014) "Oral Lichenoid Tissue Reactions: Diagnosis and Classification." Expert Review of Molecular Diagnostics 14.2: 169-184. 
10. Kyung-Eun, Lee (2016) "Oral Lichen Planus and Oral Lichenoid Lesion: Diagnosis and Assessment of Direct Immunofluorescence." Journal of Oral Medicine and Pain 41.3: 91-98.

11. Larsen, Kristine Roen, Jeanne Duus Johansen, Jesper Reibel, Claus Zachariae (2017) "Serum Cytokine Profile and Clinicopathological Findings in Oral Lichen Planus, Oral Lichenoid Lesions and Stomatitis." Clinical and Experimental Dental Research 3.6: 220-26.

12. Lin, Sun, Wu, and Chiang (1992) "Presence of Anti-basal Cell Antibodies in Oral Lichen Planus." Journal of the American Academy of Dermatology 26.6 : 943-47.

13. Müller, Susan (2017) "Oral Lichenoid Lesions: Distinguishing the Benign from the Deadly." Modern Pathology 30.

14. Ohshima, Noboru, Akira Shirai, and Akihiko Asahina (2011) "Coexistence of Linear Lichen Planus and Psoriasis in a Single Patient." Journal of Dermatology 38.12: 1182-184.

15. Raghu AR and N N Rao (2001) "Immunofluorescence in Oral Lichen Planus and Oral Lichenoid Reaction. A Review." Indian Journal of Dental Research: Official Publication of Indian Society for Dental Research 12.1: 29-34.

16. Shirasuna, Kanemitsu (2014) "Oral Lichen Planus: Malignant Potential and Diagnosis." Oral Science International 11.1: 1-7.

17. Siponen, Huuskonen, Läärä, and Salo (2010) "Association of Oral Lichen Planus with Thyroid Disease in a Finnish Population: A Retrospective Case-control Study." Oral Surgery, Oral Medicine, Oral Pathology, Oral Radiology and Endodontology 110.3: 319-324.

18. Thirumal Raj A (2017) "Oral Lichenoid Dysplasia and Exophytic Oral Verrucous Hyperplasia: Mythical Entities of Oral Pathology. " OralOncology 70:75.

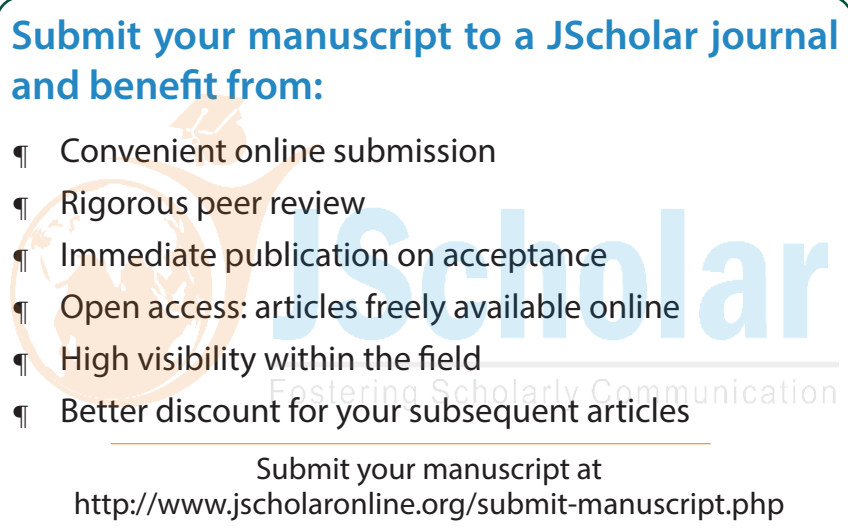

